\title{
Influence of the Thickness of a Layer of Potassium Fluoride Incorporated in the CIGS/CdS Interface on the Macroscopic Electrical Parameters of the Solar Cell
}

\author{
Djimba Niane ${ }^{1}$, Mouhamadou M. Soce ${ }^{1}$, Jean Jude Domingo ${ }^{1}$, Ousmane Diagne ${ }^{1} \&$ Moustapha Dieng ${ }^{1}$ \\ ${ }^{1}$ Laboratory of Semiconductors and Solar Energy, Physics Department, Faculty of Science and Technology, \\ University Cheikh Anta Diop, Dakar, Senegal \\ Correspondence: Djimba Niane, Laboratory of Semiconductors and Solar Energy, Physics Department, Faculty of \\ Science and Technology, University Cheikh Anta Diop, Dakar, Senegal. E-mail: djimbasbn@gmail.com
}

Received: October 27, 2018

Accepted: November 22, 2018

Online Published: January 29, 2018

doi:10.5539/apr.v11n1p1

URL: https://doi.org/10.5539/apr.v11n1p1

\begin{abstract}
In this work, the heterojunction composed of a n-type $\mathrm{ZnO}$ transparent conductive oxide (OTC) layer, a n-type $\mathrm{CdS}$ buffer layer and a absorber layer based $\mathrm{Cu}(\mathrm{In}, \mathrm{Ga}) \mathrm{Se}_{2} \mathrm{p}$ doped is studied under the influence of a KF layer placed in the CIGS/CdS interface. This study was done by varying the thickness of KF using thin-film simulation software named SCAPS-1D. The presence of KF for a doping of the CIGS absorber of $10^{16} \mathrm{~cm}^{-3}$ improves strongly the electrical parameters that are the Vco, the Jcc the FF, the maximum power and the conversion efficiency of the solar cell $\eta$. However, a decrease of the FF and the Jcc is noticed when the thickness of the KF is greater than $30 \mathrm{~nm}$ causing a deterioration of the performances of the cell.
\end{abstract}

Keywords: Solar Cells, Thin-Film, CIGS, KF, Macroscopic Electrical Parameters, Simulation

\section{Introduction}

During the past three decades, CIGS-based solar cells have been widely studied and significant progresses have been realized leading to an increasing development of this technology. Various record yields have been reported in the literature in recent years: EMPA: $20.4 \%$ ( Chirilă, A. and al., 2013), ZSW: 20.8\% (Jackson, P. and al., 2014), Solar Frontier: 20.9\% (Kushia, K., 2014), Solibro 21.0\% (Herrmann, D. et al., 2014), 21.7\% (Jackson, P. and al., 2015 ) and $22.7 \%$ (Jackson, P. and al., 2016). The use of potassium fluoride allowed the optimization of the efficiency of the solar cell. Studies on the beneficial effect of potassium fluoride on the generation and recombination of carriers in this type of cell have already been published (Niane, D. and al., \& Niane, D. and al., 2018). In this work, a numerical simulation study of the influence of the thickness of the potassium fluoride layer is made on the macroscopic electrical parameters of the solar cell.

\section{Numerical Modelling}

Most of the studies on CIGS-based solar cells are experimental, to the detriment of theoretical research which is still insufficient. However, the calculation remains a simple and economical method and it saves us the effort and the time to optimize the parameters of the solar cells in thin layers. In this paper, the study of the influence of potassium fluoride on the macroscopic electrical parameters of the CIGS-based solar cell is done with the SCAPS-1D software. It is a software that resolves the fundamental semiconductor equations (Burgelman, M. and al., 2000 \& Burgelman, M. and al., 2013).

$$
\begin{gathered}
\nabla \cdot \varepsilon \nabla \varphi=-\mathrm{q}\left(\mathrm{p}-\mathrm{n}+\mathrm{N}_{\mathrm{D}^{+}}-\mathrm{N}_{\mathrm{A}^{-}}\right) \\
\nabla \cdot \overrightarrow{J_{n}}=q(R-G)+q \frac{\partial_{n}}{\partial_{t}} \\
\nabla \cdot \overrightarrow{J_{p}}=\mathrm{q}(\mathrm{R}-\mathrm{G})+\mathrm{q} \frac{\partial_{\mathrm{p}}}{\partial_{\mathrm{t}}}
\end{gathered}
$$


Where $\varepsilon$ is the dielectric constant, $\varphi$ the electrostatic potential, $\mathrm{n}$ and $\mathrm{p}$ the concentration of the free carriers, $N_{D^{+}}$ and $N_{A^{-}}$are the densities of the ionized acceptors and donors, $J_{n}$ and $J_{p}$ the current densities of electrons and holes, $\mathrm{R}$ the rate of recombination and $\mathrm{G}$ the generation rate of electron-hole.

The KF layer used in this work at a band gap of $1.5 \mathrm{eV}$ taken from the experimental data provided in $\mathrm{O}^{\prime} \mathrm{Bryan}$ and Skinner's work, cited by Mott and Gurney, its electronic affinity $4.14 \mathrm{eV}$ and its dielectric constant $6.05 \mathrm{eV}$ (Mott, N. F., \& Gurney, R. W. 1964). The KF layer is placed between the CIGS absorbing layer and the CdS buffer layer. The structure of the $\mathrm{n}-\mathrm{ZnO} / \mathrm{n}-\mathrm{CdS} / \mathrm{p}-\mathrm{CIGS}$ heterojunction configured in the software is shown in Figure 1.

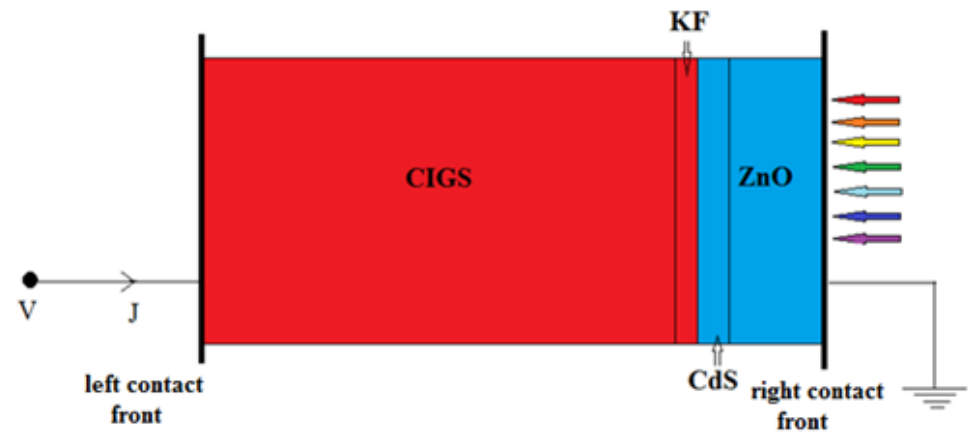

Figure 1. Structure of a heterojunction solar cell n-ZnO/n-CdS/p-CIGS

The basic parameters of the four cell layers used in the simulation are shown in Table 1.

Table 1. The basic parameters of the different layers of the cell used in the simulation: (a) and (d) denote the acceptor and donor states respectively

\begin{tabular}{llllll}
\hline & \multicolumn{5}{c}{ Layer properties } \\
& CIGS & KF & CdS & ZnO & Al-ZnO \\
\hline $\mathbf{W}(\boldsymbol{\mu m})$ & 2,5 & - & 0,05 & 0,05 & 0,2 \\
$\mathbf{E g}(\mathbf{e V})$ & 1,2 & 1,5 & 2,4 & 3,3 & 3,6 \\
$\chi(\mathbf{e V})$ & 4,5 & 4,17 & 4,2 & 4,45 & 4,45 \\
$\mathbf{\varepsilon} / \mathbf{\varepsilon}_{\mathbf{0}}$ & 13,6 & 6,05 & 10 & 9 & 9 \\
$\mathbf{N}_{\mathbf{c}}\left(\mathbf{c m}^{-3}\right)$ & $2,2.10^{18}$ & $2,2.10^{18}$ & $2,2.10^{18}$ & $2,2.10^{18}$ & $2,2.10^{18}$ \\
$\mathbf{N}_{\mathbf{v}}\left(\mathbf{c m}^{-3}\right)$ & $1,8.10^{19}$ & $1,8.10^{19}$ & $1,8.10^{19}$ & $1,8.10^{19}$ & $1,8.10^{19}$ \\
$\boldsymbol{\mu}_{\mathbf{n}}(\mathbf{c m} / \mathbf{s})$ & 100 & 100 & 100 & 100 & 100 \\
$\boldsymbol{\mu}_{\mathbf{p}}(\mathbf{c m} / \mathbf{s})$ & 25 & 25 & 25 & 25 & 25 \\
$\mathbf{N}_{\text {dopage }}\left(\mathbf{c m}^{-3}\right)$ & $1.10^{16}(\mathrm{a})$ & $1.10^{12}(\mathrm{a})$ & $1.10^{18}(\mathrm{~d})$ & $1.10^{18}(\mathrm{~d})$ & $1.10^{20}(\mathrm{~d})$ \\
\hline
\end{tabular}

\section{Results and Discussions}

The most important parameter of a solar cell is its efficiency $\eta$. It is defined as the ratio of the maximum electrical power to the power delivered by the cell of the power density irradiated by the light source. The parameters characteristic of the cells which are usually used in to describe the performance of the cell more are open-circuit voltage (Vco), the density of short-circuit current (Jcc) and the factor of form (FF).

The current-voltage density $(\mathrm{J}-\mathrm{V})$ characteristics of solar cells under illumination can be described by the phenomenological equation of the diode (Archer, M. D., 2001).

$$
\mathrm{J}_{(\mathrm{V})}=\mathrm{J}_{0}\left(\mathrm{e}^{\frac{\mathrm{q}(\mathrm{V}-\mathrm{RsJ})}{\mathrm{AkT}}}-1\right)+\frac{\mathrm{V}-\mathrm{RsJ}}{\mathrm{Rp}}-\mathrm{J}_{\mathrm{ph}}
$$

where $\mathrm{J}$ is the current density, $\mathrm{V}$ is the applied bias voltage, $\mathrm{J}_{0}$ is a pre-factor named saturation current density, q is the charge of the electron, Rs and Rp are the series and parallel resistors respectively, A is the ideal factor of the diode, $\mathrm{kT}$ is the Boltzmann constant multiplied by the absolute temperature, and Jph is the current generated by light.

The equation of the diode (4) contains photocurrents of independent polarization, it is ideal for example when the diffusion lengths of the minority carriers are very small. In the absence of series resistance and shunts (ie Rs $=0$ and $\mathrm{Rp}=\infty$ ), the equation of the diode is simplified: 


$$
\mathrm{J}_{(\mathrm{V})}=\mathrm{J}_{0}\left(\mathrm{e}^{\frac{\mathrm{qV}}{\mathrm{AkT}}}-1\right)-\mathrm{J}_{\mathrm{ph}}
$$

Thus, the following expressions for the short circuit current Jcc $(\mathrm{V}=0)$ and the open circuit voltage Vco $(\mathrm{Jcc}=0)$ are obtained:

$$
\begin{gathered}
\mathrm{J}_{\mathrm{cc}}=-\mathrm{J}_{\mathrm{ph}} \\
\mathrm{V}_{\mathrm{co}}=\frac{\mathrm{AkT}}{\mathrm{q}} \ln \left(\frac{\mathrm{J}_{\mathrm{ph}}}{\mathrm{J}_{0}}+1\right)
\end{gathered}
$$

The efficiency of the cell is calculated from the ratio of the maximal power density delivered by the cell $(\mathrm{JmVm})$ to the irradiated power density Ps:

$$
\eta=\frac{\mathrm{Im}_{\mathrm{m}} \mathrm{V}_{\mathrm{m}}}{\mathrm{P}_{\mathrm{S}}}=\frac{\mathrm{ICC}_{\mathrm{CO}} \mathrm{FF}}{\mathrm{P}_{\mathrm{S}}}
$$

where the form factor FF is defined as:

$$
\mathrm{FF}=\frac{\mathrm{Jm}_{\mathrm{m}} V_{\mathrm{m}}}{\mathrm{JcC}_{\mathrm{CCO}}}
$$

\subsection{Characteristic $J(V)$}

Figure 2 shows the characteristic J (V) in the case with and without presence of KF. Simulations are made taking $15 \mathrm{~nm}$ of KF and the doping rate of the CIGS layer was set at $N_{b}=10^{16} \mathrm{~cm}^{-3}$ at room temperature $25^{\circ} \mathrm{C}$.

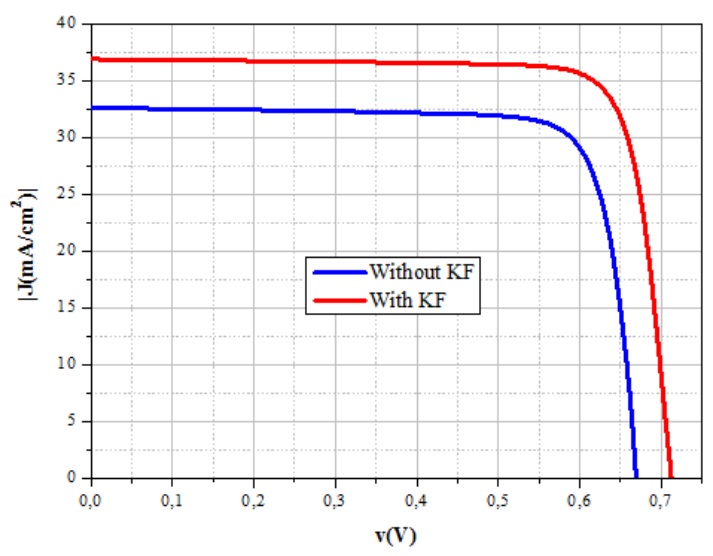

Figure 2. Characteristic $\mathrm{J}(\mathrm{V})$ Without $\mathrm{KF}$ and With KF

The current-voltage characteristic shows that the presence of potassium fluoride (KF) causes an increase of the open-circuit voltage and the short-circuit density. Electrical output parameters such as open circuit voltage, short circuit current density, maximum photocell power, form factor and cell efficiency are determined from the J-V characteristics. In addition, it was noted during the simulation that all its parameters improve with the presence of KF in the solar cell.

Table 2 compared the different values obtained from one cell with KF and another cell without KF.

Table 2. Comparison of the electrical parameters of a cell without KF and another cell with KF

\begin{tabular}{llllll}
\hline Parameters & $\mathrm{V} c \mathrm{~V}(\mathrm{~V})$ & $\mathrm{Jcc}\left(\mathrm{mA} \cdot \mathrm{cm}^{-2}\right)$ & $\mathrm{FF}(\%)$ & $\mathrm{Pm}\left(\mathrm{mW} . \mathrm{cm}^{-2}\right)$ & $\eta(\%)$ \\
\hline Cell without KF & 0,669034 & 32,63661830 & 81,4666 & 17,7882425 & 18,5762 \\
Cell with KF & 0,712446 & 36,90477082 & 82,5314 & 21,6996856 & 22,6609 \\
\hline
\end{tabular}

The table confirms that the solar cell in the presence of KF gives better performance. The efficiency of the cell went from $18.5762 \%$ for a cell without KF to $22.6609 \%$ for a cell with KF. We estimate the increase at $4.18 \%$. This could be attributed to the beneficial effects of KF on the parameters whose efficiency depends. In what follows, we will study the effect of the KF layer thickness on the electrical macroscopic parameters of the cells that are generally used to describe the performance of the cell. 


\subsection{Short-Circuit Current Density (Jcc)}

In Figure 3, the short-circuit density is represented as a function of the thickness of the KF layer.

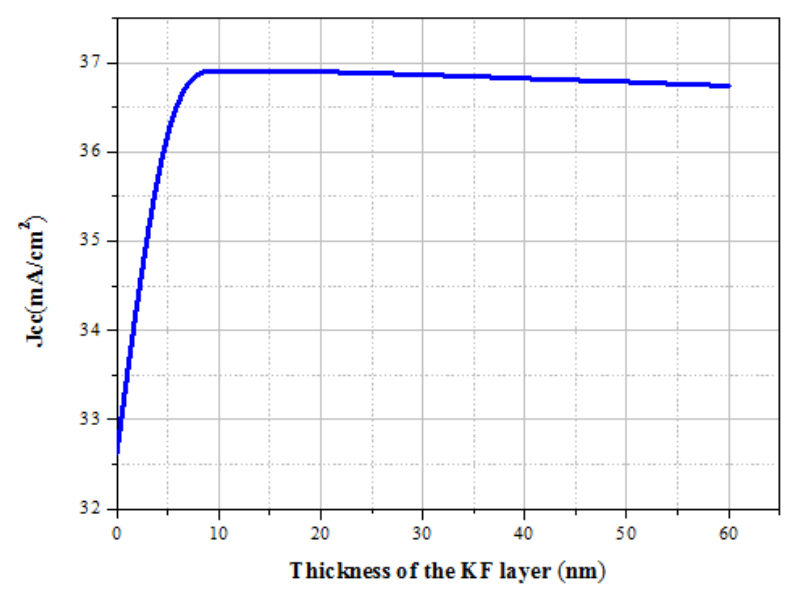

Figure 3. Short-circuit current density as a function of the thickness of the KF layer

The profile of the short-circuit current density shows an increase as a function of the thickness of the KF layer within the photocell. This increase is more pronounced when the thickness is between 0 and $10 \mathrm{~nm}$. Then, this maximum value of Jcc becomes constant when the thickness is between $10 \mathrm{~nm}$ and $25 \mathrm{~nm}$ and finally beyond $25 \mathrm{~nm}$ a slight decrease in the value of Jcc is noticed. Improvement of Jcc with the presence of the KF layer is caused by passivation of the CIGS/CdS interface. This results in a high probability that carriers can be collected in the cell.

\subsection{Open Circuit Voltage}

The effect of the thickness of the KF layer on the open circuit voltage is shown in Figure 4.

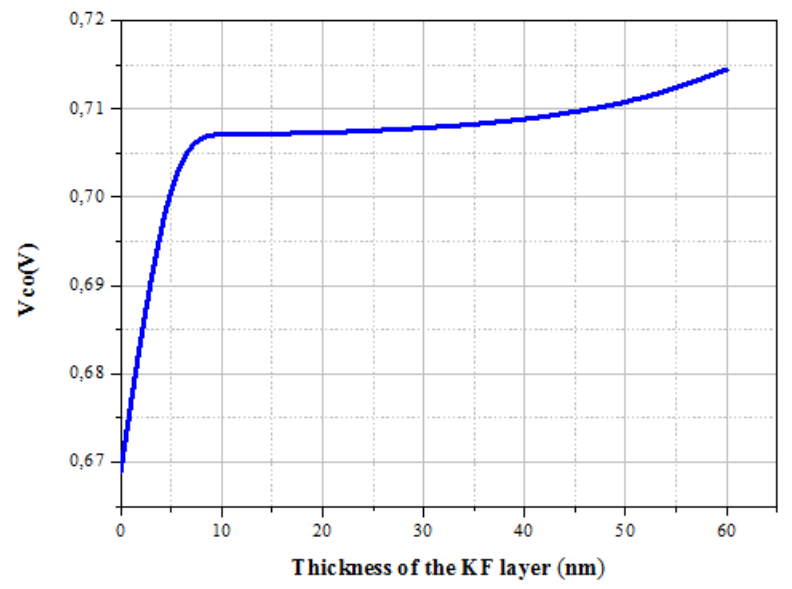

Figure 4. Open circuit voltage as a function of the thickness of the KF layer

We notice that the open circuit voltage increases with increasing content of the KF layer in the CIGS/CdS interface. The behavior of the Vco can be attributed to the improvement of the band gap which causes a narrowing of the space charge area and the decrease of the saturation current density. Indeed, a large amount of $\mathrm{KF}$ in the interface makes it possible to passivate the grain boundaries and compensate for donor-type defects. This results a strong carrier recombination barrier in the $\mathrm{n}-\mathrm{ZnO} / \mathrm{n}-\mathrm{CdS} / \mathrm{p}-\mathrm{CIGS}$ heterojunction.

\subsection{Maximal Power}

The Figure 5 illustrates the effect of the thickness of KF on the maximum power of the solar cell.

We note an increase of the power with the increase of the thickness of KF up to an optimal zone between $8 \mathrm{~nm}$ and $25 \mathrm{~nm}$. Then, the power decreases gradually when the thickness exceeds $25 \mathrm{~nm}$. This decrease is due to the losses of current generated by the solar cell. 


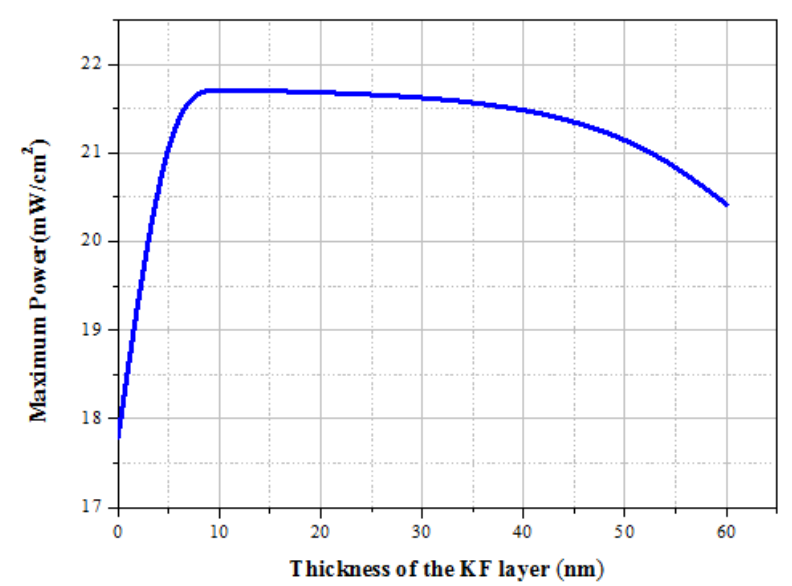

Figure 5. Maximum power as a function of the thickness of the KF layer

\subsection{Form Factor}

The profile of the form factor as a function of the thickness of the KF layer is shown in Figure 6.

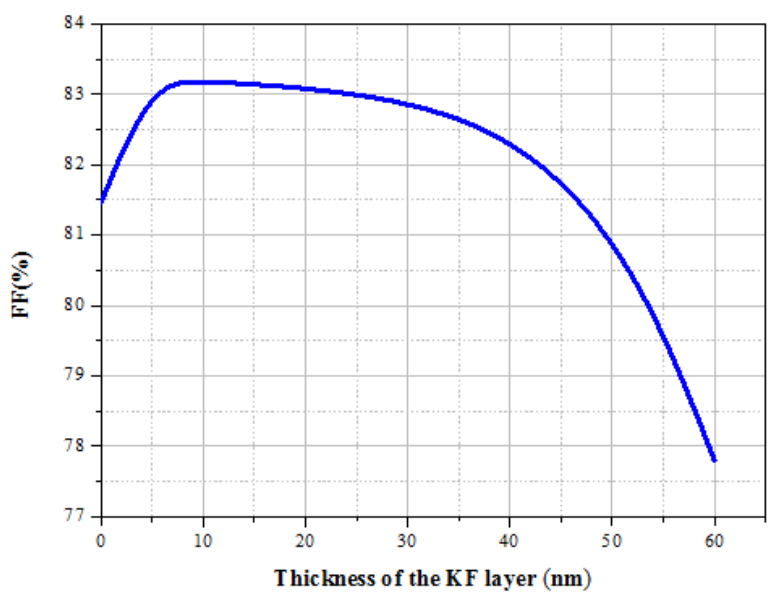

Figure 6. Form factor as a function of the thickness of the KF layer

We find that the form factor is influenced by the increase of the KF layer in the CIGS/CdS interface. Indeed, an improvement of the form factor is observable up to a thickness of KF of around 10nm corresponding to a maximum value of $83.18 \%$, which then follows a deterioration of this parameter. The deterioration of the cell form factor is related to the loss factors noted in the short-circuit current density and the maximum power while its improvement can be attributed to the inter-diffusion of $\mathrm{K}$ in the CIGS.

\subsection{Cell Efficiency}

Figure 7 illustrates the shape of the efficiency of the cell $\eta$ as a function of the thickness of the KF layer.

We note an increase in the conversion efficiency of the cell with the thickness of the KF layer. However, a gradual decrease of $\eta$ is noticed when the amount of KF exceeds $25 \mathrm{~nm}$. The additional gain in efficiency can be attributed to the improved passivation of the CIGS/CdS interface which is caused by a lowering of the valence band maximum compared to the Fermi energy level and on the other hand by the increase of Vco and FF. Moreover, we can say in accordance with the yields found in the literature that the optimal thickness of KF must be taken between $10 \mathrm{~nm}$ and $30 \mathrm{~nm}$ not to damage the solar cell. 


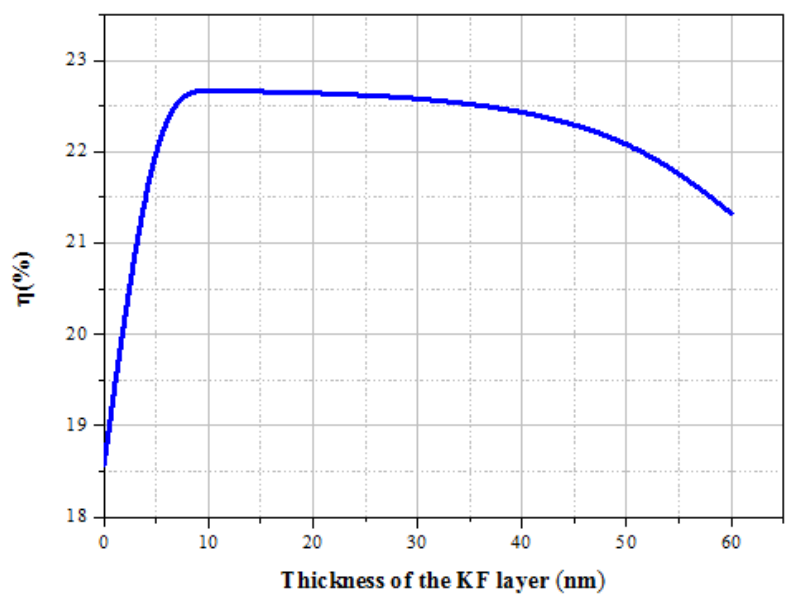

Figure 7. Efficiency of the cell $\eta$ as a function of the thickness of the KF layer

\subsection{External Quantum Efficiency}

The external quantum efficiency noted $(\mathrm{QE})$, represents the ratio of the number of charges collected on the number of incident photons as a function of the wavelength. These measurements are used to characterize the photocurrent of cells and determine the origin of losses Jcc, his expression is given by (Niane, D. and al., 2016):

$$
\mathrm{QE}(\lambda)=\frac{\mathrm{J}(\lambda)}{\mathrm{q} \times \varphi(\lambda)}
$$

Whit $J(\lambda)$ the current collected at one hundred wavelength $\lambda$, q the elementary charge, and $\varphi(\lambda)$ the photon flux at the chosen wavelength.

The quantum yield depends on several properties of the material such as the absorption coefficient, the junction depth, the thickness of the space charge zone, the lifetime of the carriers and their mobility but also the surface recombination.

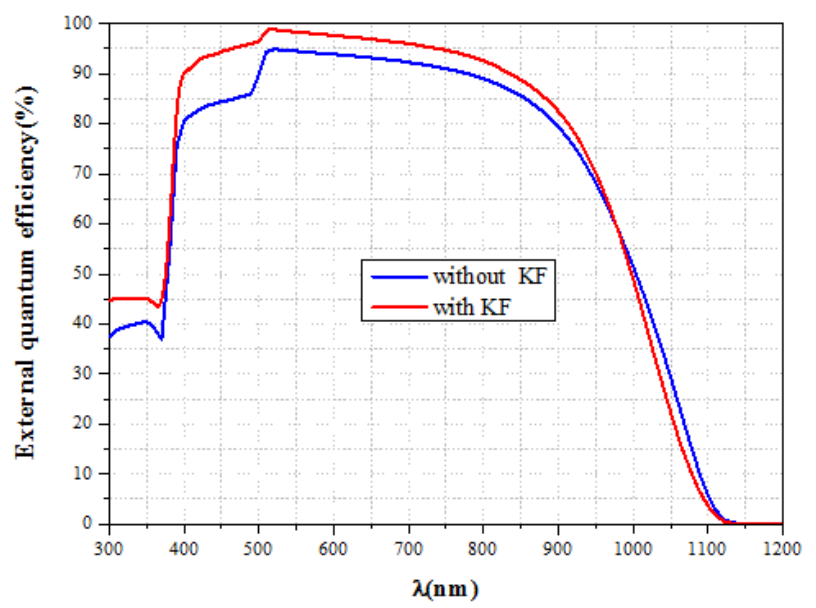

Figure 8. External quantum efficiency as a function of the wavelength $(\lambda)$ of a solar cell with KF and another cell without KF

The quantum efficiency of a solar cell as a function of the wavelength of the incident light is called the spectral response. Figure 8 shows the variation of the quantum efficiency as a function of the wavelength of a solar cell with $\mathrm{KF}$ and another cell without KF. From 300 to $400 \mathrm{~nm}$ and from 400 to $500 \mathrm{~nm}$ corresponding respectively to the absorption zone of the $\mathrm{ZnO}$ window layer and that of the buffer layer $\mathrm{CdS}$, we see clearly that the presence of $\mathrm{KF}$ in the CIGS/CdS interface results a more important quantum efficiency. Beyond $550 \mathrm{~nm}$ corresponding to the absorption zone of the absorbing layer CIGS, the external quantum efficiency continues to increase with the presence of KF reaching a maximum corresponding to $98.5 \%$, but nevertheless decreases with increasing lengths wave. This can be explained by the fact that the CIGS has a direct gap, the absorption in the heterojunction is greater in the base, and it improves when the KF layer is put in the interface CIGS/CdS. Indeed, the presence of 
the KF layer reduces the width of the band gap and multiplies the phonons. The mechanism of production of the minority carriers will dominate the recombination mechanism in this wavelength range, thus leading to the increase of the current density, hence the quantum efficiency. However, a reversal of the curves is noted for wavelengths greater than $1000 \mathrm{~nm}$.

\section{Conclusion}

Potassium fluoride has a very important role in the passivation of the CIGS/CdS interface. In this work, we studied the influence of the KF content on the parameters of a $\mathrm{n}-\mathrm{ZnO} / \mathrm{n}$-CdS/p-CIGS heterojunction solar cell. The conversion efficiency of the cell increased from $18.5762 \%$ for a cell without KF to $22.6609 \%$ for a cell with KF. However, increasing the thickness of the KF layer beyond $30 \mathrm{~nm}$ showed a deterioration of parameters such as form factor and current density as well as conversion efficiency. This suggests that the optimal range for a better performance of the cell must be between [10nm, 30nm].

\section{Conflict of interests}

The authors declare that there is no conflict of interests regarding the publication of this paper.

\section{References}

Archer, M. D. (2001). The past and present. In M. D. Archer \& R. Hill (Eds.), Clean Electricity from Photovoltaics (pp. 1-32). London: Imperial College Press.

Burgelman, M., Decock, K., Khelifi, S., \& Abass, A. (2013). Advanced electrical simulation of thin film solar cells. Thin Solid Films, 535, 296-30.

Burgelman, M., Nollet, P., \& Degrave, S. (2000). Modelling polycrystalline semiconductor solar cells. Thin Solid Films, 361/362, 527-532.

Chirilă, A., Reinhard, P., Pianezzi, F., Bloesch, P., .., \& Tiwari, A. N. (2013). Nature Mater, 12, 1107.

Herrmann, D. et al. (2014, June 8-13). CIGS module manufacturing with high deposition rates and efficiencies. Presented at 40th IEEE PVSC, Denver, CO, USA.

Jackson, P., Hariskos, D., Wuerz, R., Kiowski, O., .., \& Powalla, M. (2015, January). Properties of $\mathrm{Cu}(\mathrm{In}, \mathrm{Ga}) \mathrm{Se}_{2}$ solar cells with new record efficiencies up to $21.7 \%$. Phys. Status Solidi RRL-Rapid Res. Lett., 9(1), 28-31.

Jackson, P., Hariskos, D., Wuerz, R., Wischmann, W., \& Powalla, M. (2014). Phys. Status Solidi RRL, 8, 219.

Jackson, P., Wuerz, R., Hariskos, D., Lotter, E., ..., \& Powalla, M. (2016). Effects of heavy alkali elements in $\mathrm{Cu}(\mathrm{In}, \mathrm{Ga}) \mathrm{Se}_{2}$ solar cells with efficiencies up to 22.6\%. Phys. Status Solidi RRL, 10(8), 583-586.

Kushia, K. (2014, April 2-3). Current Status and Future Prospects of Solar Frontier K.K.. Presented at the IW-CIGS Tech 5, Berlin, Germany.

Mott, N. F., \& Gurney, R. W. (1964). Electronic Processes in Ionic Crystals (p. 274). Dover, New York.

Niane, D., Diagne, O., Ehemba, A. K., Soce, M. N., \& Dieng, M. (2018). Generation and Recombination of a CIGSe Solar Cell under the Influence of the Thickness of a Potassium Fluoride (KF) Layer. American Journal of Materials Science and Engineering, 6(2), 26-30.

Niane, D., Ehemba, A. K., Cissé, S., Diagne, O., \& Dieng, M. (2018). Influence of the Doping Rate of the CIGSe Layer on the Recombination-generation Mechanisms with $25 \mathrm{~nm}$ Incorporation of a KF Layer on a CIGSe Solar Cell. American Journal of Energy Research, 6(1), 30-34.

Niane, D., Ehemba, A. K., Diallo, D., Wade, I., \& Dieng, M. (2016). The Influence of Temperature on the Electric Parameters of a Solar Cell Based on $\mathrm{Cu}(\mathrm{In}, \mathrm{Ga}) \mathrm{Se}_{2}$. International Journal of Scientific Engineering and Technology, (5), 247-251.

\section{Copyrights}

Copyright for this article is retained by the author(s), with first publication rights granted to the journal.

This is an open-access article distributed under the terms and conditions of the Creative Commons Attribution license (http://creativecommons.org/licenses/by/4.0/). 